\title{
An Exact Method for the Analysis of a Two-Machine Manufacturing System with a Finite Buffer Subject to Time-Dependent Failure
}

\author{
Beixin Xia, Jianping Chen, and Zaifang Zhang \\ School of Mechatronics Engineering and Automation, Shanghai University, Shanghai 200072, China \\ Correspondence should be addressed to Zaifang Zhang; zaifangzhang@shu.edu.cn
}

Received 27 March 2015; Revised 2 June 2015; Accepted 3 June 2015

Academic Editor: Qing (Cindy) Chang

Copyright (c) 2015 Beixin Xia et al. This is an open access article distributed under the Creative Commons Attribution License, which permits unrestricted use, distribution, and reproduction in any medium, provided the original work is properly cited.

\begin{abstract}
This paper presents an efficient exact analytical method for evaluating the performance of a two-machine manufacturing system with a finite buffer. Unlike existing work, it is assumed that the buffer is prone to time-dependent failure, that is, failure that can occur even when the buffer is not working. First, Markov model is established for the system. Then transition equations are derived based on the system state analysis. After that, a solution technique is provided to obtain the results. Finally, numerical cases are carried out to explore the internal laws of the system. The relationships between system parameters and system performance are investigated. Furthermore, the difference between buffer subject to time-dependent failure and buffer subject to operation-dependent failure is discussed. The proposed method is the building block of approximate analytical methods which can greatly improve the accuracy when analyzing long systems.
\end{abstract}

\section{Introduction and Related Work}

In an ideal manufacturing system, the machines have the same cycle time and never break down. Parts flow through the system fluently and steadily. However, in real systems this ideal manufacturing process could be disrupted by the fluctuation of cycle times or machine failures. For instance, machines may vary a lot in their types and thus have different cycle times; the cycle time of a manual operation is not deterministic and may obey some random distribution; machines may be prone to failure and need to stop for maintenance. All the situations above can penalize the productivity of the system and increase losses in availability for the whole plant [1].

In order to cope with these situations, buffers are placed between machines which can highly improve the efficiency of the system. As a typical type of buffer, accumulating conveyor has been widely used in actual manufacturing systems especially in automated production lines. It provides storage and buffering between two consecutive machines. In practice, accumulating conveyors have finite buffer capacities. Since accumulating conveyors are mechanical devices, they are prone to failure. There are two types of failures, referred to as operation-dependent failure and time-dependent failure [2]. An operation-dependent failure can occur only while the device is working while a time-dependent failure can occur even when the device is forced down [3]. For accumulating conveyors, the failure they are prone to is assumed to be timedependent rather than operation-dependent. It is due to the fact that accumulating conveyors run continuously, not only when parts are being loaded or unloaded, which means they are subject to failure even when the adjacent machines are not loading or unloading a part from the conveyor [4].

When configuring a system with buffers like accumulating conveyors, how to determine the parameters of those buffers is a complex problem. To solve the problem, performance evaluation methods which can identify the relationships between system parameters and system performance have been developed as powerful decision support tools when designing or optimizing systems.

Lots of work has been devoted to the research of performance evaluation technologies. Generally, it can be mainly classified into simulation methods and analytical methods. Simulation methods can model a system at any required 
level of detail and provide accurate results. But they are too time consuming to be used under some circumstances, for instance, the early design stage where lots of alternative configurations need to be evaluated. On the contrary, analytical methods are much more efficient. They can evaluate systems in a short time. Although the results are approximate, they are accurate enough at the early design stage of manufacturing systems because there is often significant inaccuracy in the input data [5]. Analytical methods can be further divided into exact analytical methods and approximate analytical methods. Exact analytical methods can provide exact results but are only available for small systems. Approximate analytical methods which are mostly based on decomposition method can cope with large systems and are very practical. Nonetheless, exact analytical methods are still of significant importance since (1) exact results obtained by exact methods are better than simulations or approximations when the models fit real systems closely, (2) they provide useful qualitative insight into the behaviour of systems, and (3) the fact that they can be solved rapidly makes them essential parts of approximate methods [6].

Even though extensive research has been done on exact analytical methods, most of it has been focused on studying machine behaviour. By using an exact method based on discrete Markov model, Buzacott [7] analyzed a two-machine system in which the cycle times of the two machines are deterministic and identical and the times between failures and the times to repair of the machines are assumed to obey exponential distribution. Hillier and Boling [8] established a model by considering reliable machines with exponentially distributed or geometrically distributed cycle times. In Altiok's [9] model, the machines are unreliable, the cycle times and the times to repair obey phase type distributions, and the times between failures obey exponential distribution. In order to evaluate inhomogeneous systems in which the cycle times are deterministic but not identical, a continuous Markov model was developed in [10] and then extended in [11-14]. Moreover, Tolio et al. [15] proposed an analytical method for performance evaluation of two-machine lines. In that model, the machines have multiple failure modes and can fail in different ways. Several researchers aim at establishing a general model for two-machine systems. Tan and Gershwin [16] investigated general Markovian continuous-flow production systems and developed a methodology that can handle a wide range of systems as long as the transition rates and the flows rates were provided. Tan and Gershwin [17] illustrated the methodology by analyzing various systems studied in the previous literature. Other types of systems also have been analyzed. Li et al. [18] gave out a Markov method for the analysis of quality-caused reentrance electrical test system. Gebennini and Gershwin [19] modelled two-machine transfer lines taking into account waste production. Gebennini et al. $[20,21]$ developed a discrete time model and a continuous time model with restart policy, respectively. However, all of the work above is under a simple assumption that buffers are finite. It does not fit real manufacturing systems in a lot of cases. Some other behaviours about buffer, such as buffer failures, are still unclear and deserve more investigations [22].
Buffer failures affect system performance significantly. Lipset et al. [4] illustrated a statistical report from US car manufacturing plants, according to which $27.7 \%$ of all system down time was caused by accumulating conveyor breakdowns. On the contrary, the study on systems with unreliable buffers has not got enough attention. A discrete Markov model was generated and analyzed by Burman [23]. In that model, the system is homogeneous and the buffers are unreliable and prone to operation-dependent failure. Transporter is a representative of this type of buffers. A transporter breaks down only when parts are being loaded. Later, an inhomogeneous system with a buffer subject to operation-dependent failure was modelled as a continuous Markov process [24]. Liu et al. [25] introduced machines' multiple failure modes into Burman's model and presented a more general method. Unfortunately, to the best of our knowledge, no exact analytical method has been provided for systems with buffers subject to time-dependent failure, like accumulating conveyors. Lipset et al. [4] analyzed twomachine systems with this type of buffer and developed an approximate analytical method. The maximal error of examples presented in that paper is about $3.1 \%$. Based on that method, Lipset et al. [26] and Lipset et al. [27] proposed decomposition methods for performance evaluation of longer production lines. Although the errors in [4] are minor, as the method for the analysis of two-machine systems is the building block of approximate analytical methods for the analysis of large systems, those minor errors will be enlarged and result in huge errors finally.

The purpose of this paper is to develop an exact analytical method for two-machine systems with buffers like accumulating conveyors by using Markov model. Based on this method, large systems with buffers subject to time-dependent failure can be evaluated more accurately.

The remaining part of the paper is organized as follows. A detailed formulation of the problem is shown in Section 2. Markov model is established and analyzed in Section 3. Buffer reliability effects and comparisons between buffer subject to time-dependent failure and buffer subject to operationdependent failure are illustrated in Section 4. Finally, conclusions and future work are present in Section 5.

\section{Problem Formulation}

The two-machine manufacturing system studied in this paper consists of machine $M_{1}$, machine $M_{2}$, and buffer $B_{1}$ which separated the two machines. Parts are transferred from outside the system to machine $M_{1}$, then to buffer $B_{1}$, and then to machine $M_{2}$, after which they leave the system.

\subsection{Assumptions. The following assumptions are made:}

(i) The system is homogeneous which means the cycle times of machine $M_{1}$ and $M_{2}$ are identical. To simplify the model, the cycle times are scaled to 1 time unit.

(ii) Both of the machines are unreliable. The failures are operation-dependent. The times between failures and 
the times to repair of machine $M_{i}$ are geometrically distributed with parameters $p_{i}$ and $r_{i}$, respectively.

(iii) Buffer $B_{1}$ has finite capacity $N_{1}$. It is unreliable and prone to time-dependent failure. The times between failures and the times to repair of buffer $B_{1}$ are geometrically distributed with parameters $p_{b}$ and $r_{b}$, respectively.

(iv) Machine $M_{1}$ is blocked if buffer $B_{1}$ breaks down or buffer $B_{1}$ is operational and full but machine $M_{2}$ fails. It is assumed that there are infinite parts before machine $M_{1}$. Hence, machine $M_{1}$ is never starved.

(v) Machine $M_{2}$ is starved if buffer $B_{1}$ breaks down or buffer $B_{1}$ is operational and empty but machine $M_{1}$ fails. It is assumed that there is infinite storage space after machine $M_{2}$. Hence, machine $M_{2}$ is never blocked.

2.2. System Dynamic. Let $\alpha_{i}(t)$ and $\alpha_{b}(t)$ denote the state of machine $M_{i}$ and buffer $B_{1}$ at time $t$, respectively. We have

$$
\begin{aligned}
& \alpha_{i}(t)= \begin{cases}1, & \text { when machine } M_{i} \text { is operational, } \\
0, & \text { when machine } M_{i} \text { is down, }\end{cases} \\
& \alpha_{b}(t)= \begin{cases}1, & \text { when buffer } B_{1} \text { is operaional, } \\
0, & \text { when buffer } B_{1} \text { is down. }\end{cases}
\end{aligned}
$$

To be noticed, the fact that machine $M_{i}$ is operational means either it is working or it is idle due to starvation or blockage while the fact that buffer $B_{1}$ is operational only means it is working.

Let $n(t)$ denote the part number in buffer $B_{1}$ at time $t$. Then, the system state $s(t)$ at time $t$ is given by

$$
s(t)=\left(n(t), \alpha_{1}(t), \alpha_{2}(t), \alpha_{b}(t)\right) .
$$

According to the assumptions, if machine $M_{i}$ is working at time $t$ there is a constant probability $p_{i}$ that machine $M_{i}$ fails at time $t+1$. Hence, the transition probability

$$
\operatorname{prob}\left[\alpha_{i}(t+1)=0 \mid \alpha_{i}(t)=1\right]=p_{i} .
$$

Similarly, we have

$$
\begin{aligned}
& \operatorname{prob}\left[\alpha_{i}(t+1)=1 \mid \alpha_{i}(t)=1\right]=1-p_{i}, \\
& \operatorname{prob}\left[\alpha_{i}(t+1)=1 \mid \alpha_{i}(t)=0\right]=r_{1}, \\
& \operatorname{prob}\left[\alpha_{i}(t+1)=0 \mid \alpha_{i}(t)=0\right]=1-r_{1} .
\end{aligned}
$$

For a buffer subject to operation-dependent failure, it cannot fail when there is no part in it. On the contrary, as a buffer subject to time-dependent failure, buffer $B_{1}$ could break down regardless of the part number in it. According to that, we have

$$
\begin{aligned}
& \operatorname{prob}\left[\alpha_{b}(t+1)=0 \mid \alpha_{b}(t)=1\right]=p_{b}, \\
& \operatorname{prob}\left[\alpha_{b}(t+1)=1 \mid \alpha_{b}(t)=1\right]=1-p_{b}, \\
& \operatorname{prob}\left[\alpha_{b}(t+1)=1 \mid \alpha_{b}(t)=0\right]=r_{b}, \\
& \operatorname{prob}\left[\alpha_{b}(t+1)=0 \mid \alpha_{b}(t)=0\right]=1-r_{b} .
\end{aligned}
$$

As the machines are prone to operation-dependent failure, they can only fail when they are working. According to that, we have

$$
\begin{aligned}
& \operatorname{prob}\left[\alpha_{1}(t+1)=1 \mid \alpha_{1}(t)\right. \\
& \left.\quad=1,\left(\alpha_{b}(t)=0 \text { or } n(t)=N\right)\right]=1, \\
& \operatorname{prob}\left[\alpha_{1}(t+1)=0 \mid \alpha_{1}(t)\right. \\
& \left.\quad=1,\left(\alpha_{b}(t)=0 \text { or } n(t)=N\right)\right]=0, \\
& \operatorname{prob}\left[\alpha_{2}(t+1)=1 \mid \alpha_{2}(t)\right. \\
& \left.\quad=1,\left(\alpha_{b}(t)=0 \text { or } n(t)=0\right)\right]=1, \\
& \operatorname{prob}\left[\alpha_{2}(t+1)=0 \mid \alpha_{2}(t)\right. \\
& \left.\quad=1,\left(\alpha_{b}(t)=0 \text { or } n(t)=0\right)\right]=0 .
\end{aligned}
$$

As the machines cannot fail when the buffer breaks down, we have

$$
\begin{aligned}
& \operatorname{prob}\left[\alpha_{i}(t+1)=1 \mid \alpha_{i}(t)\right. \\
& \left.\quad=1,\left(\alpha_{b}(t+1)=0 \text { or } \alpha_{b}(t)=0\right)\right]=1, \\
& \operatorname{prob}\left[\alpha_{i}(t+1)=0 \mid \alpha_{i}(t)\right. \\
& \left.\quad=1,\left(\alpha_{b}(t+1)=0 \text { or } \alpha_{b}(t)=0\right)\right]=0 .
\end{aligned}
$$

Let $I_{i}(t+1)$ denote the number of parts transferred by machine $M_{i}$. It is defined as follows:

$$
\begin{aligned}
& I_{1}(t+1) \\
& \quad= \begin{cases}1, & \alpha_{1}(t+1)=1, \alpha_{b}(t+1)=1, n(t)<N, \\
0, & \text { otherwise, }\end{cases} \\
& I_{2}(t+1) \\
& \quad= \begin{cases}1, & \alpha_{2}(t+1)=1, \alpha_{b}(t+1)=1, n(t)>0, \\
0, & \text { otherwise. }\end{cases}
\end{aligned}
$$

Consequently, the buffer level at time $t+1$ is

$$
n(t+1)=n(t)+I_{1}(t+1)-I_{2}(t+1) .
$$

2.3. Performance Measures. The most important performance measures of a manufacturing system are the production rate and the average buffer level. The production rate $\mathrm{PR}_{i}$ of machine $M_{i}$ can be obtained by

$$
\begin{aligned}
& \mathrm{PR}_{1}=\operatorname{prob}\left[\alpha_{1}=1, \alpha_{b}=1, n<N\right] \\
& \mathrm{PR}_{2}=\operatorname{prob}\left[\alpha_{2}=1, \alpha_{b}=1, n>0\right] .
\end{aligned}
$$

Because flow in a steady manufacturing system is conserved [28], we have

$$
\mathrm{PR}_{1}=\mathrm{PR}_{2}=\mathrm{PR} \text {. }
$$


Let $p\left(n, \alpha_{1}, \alpha_{2}, \alpha_{b}\right)$ denote the steady probability of system state $s$ where $s=\left(n, \alpha_{1}, \alpha_{2}, \alpha_{b}\right)$. The average buffer level $\bar{n}$ can be calculated by

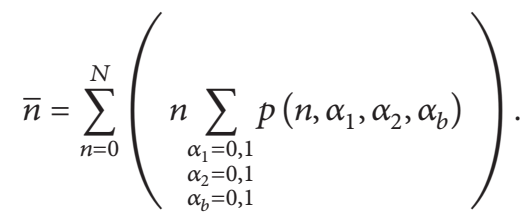

\section{Solution Methodology}

3.1. Transient States. A transient state in Markov process has no possible predecessor except itself or another transient state. The steady probability of a transient state is zero. Removing all transient states before analyzing the system can simplify the work.

The state $(0,1,0,1)$ is transient. If the system is in state $(0,1,0,1)$ at time $t+1$, from (9) and (10) we have $I_{1}(t+1)=1$ and $I_{2}(t+1)=0$. As $n(t+1)=n(t)+1, n(t+1)=0$, and $n(t) \geq 0$ it is easy to find that the state $(0,1,0,1)$ cannot be reached from other states except itself.

The state $(0,1,1,1)$ is transient. If the system is in state $(0,1,1,1), I_{1}(t+1)=1$ from (9). Furthermore, we have $n(t+$ $1)=n(t)+1-I_{2}(t+1)$. As $n(t+1)=0$, it can be inferred that $n(t)=0$ and $I_{2}(t+1)=1$. However, the results are conflicting according to (10).

The states $(0,0,0,0)$ and $(0,0,0,1)$ are transient. Assume that the system is in state $(0,0,0,0)$ at time $t+1$, and from (9), (10) and (11) we have $n(t)=0$. As the machines cannot fail when the buffer breaks down we have $\alpha_{1}(t)=0$ and $\alpha_{2}(t)=0$ based on (8). Hence, the possible previous states of $(0,0,0,0)$ are $(0,0,0,1)$ and itself. Similarly, assume that $(0,0,0,1)$ is the system state at time $t+1$; it is easy to find out that $n(t)=$ $0, \alpha_{1}(t)=0$, and $\alpha_{2}(t)=0$. The possible previous states of $(0,0,0,1)$ are $(0,0,0,0)$ and itself. Therefore, $(0,0,0,0)$ and $(0,0,0,1)$ form a closed loop. Since neither of the states can be the initial state of the system in reality, both of them are transient.

The state $(0,1,0,0)$ is transient. If the system is in state $(0,1,0,0)$ at time $t+1$, from (9), (10), and (11) we have $n(t)=0$. As $\alpha_{b}(t+1)=0$, the machines cannot break down. Hence, $\alpha_{2}(t)=0$. It turns out that all eligible states except itself are transient.

The state $(1,1,0,1)$ is transient. If the system is in state $(1,1,0,0)$ at time $t+1$, from (9), (10), and (11) we have $n(t)=0$. Since machine $M_{2}$ is starved and cannot fail, $\alpha_{2}(t)=0$. Only states of the form $\left(0, \alpha_{1}(t), 0, \alpha_{b}(t)\right)$ have to be considered. However, all of these states are transient.

Similarly, it can be deduced that $(N, 0,0,0),(N, 0,0,1)$, $(N, 0,1,0),(N, 0,1,1),(N, 1,1,1)$, and $(N-1,0,1,1)$ are all transient.

3.2. Transition Equations. After eliminating the transient states, the relationships between the steady states are analyzed. According to the number of parts in the buffer, these steady states are classified into 3 categories: (1) internal states, in which $2 \leq n \leq N-2$, (2) lower boundary states, which are related to the states with a buffer level of $n=0$, and (3) upper bounder states, which are related to the states with a buffer level of $n=N$. Corresponding transition equations are inferred as follows.

First, the internal state transitions are shown in Figure 1. The following are the internal equations:

$$
\begin{aligned}
& p(n, 0,0,0) \\
& =\left(1-r_{1}\right)\left(1-r_{2}\right)\left(1-r_{b}\right) p(n, 0,0,0) \\
& +\left(1-r_{1}\right)\left(1-r_{2}\right) p_{b} p(n, 0,0,1) \text {, } \\
& p(n, 0,0,1) \\
& =\left(1-r_{1}\right)\left(1-r_{2}\right) r_{b} p(n, 0,0,0) \\
& +\left(1-r_{1}\right)\left(1-r_{2}\right)\left(1-p_{b}\right) p(n, 0,0,1) \\
& +\left(1-r_{1}\right) p_{2}\left(1-p_{b}\right) p(n, 0,1,1) \\
& +p_{1}\left(1-r_{2}\right)\left(1-p_{b}\right) p(n, 1,0,1) \\
& +p_{1} p_{2}\left(1-p_{b}\right) p(n, 1,1,1), \\
& p(n, 0,1,0) \\
& =\left(1-r_{1}\right) r_{2}\left(1-r_{b}\right) p(n, 0,0,0) \\
& +\left(1-r_{1}\right) r_{2} p_{b} p(n, 0,0,1) \\
& +\left(1-r_{1}\right)\left(1-r_{b}\right) p(n, 0,1,0) \\
& +\left(1-r_{1}\right) p_{b} p(n, 0,1,1) \text {, } \\
& p(n, 0,1,1) \\
& =\left(1-r_{1}\right) r_{2} r_{b} p(n+1,0,0,0) \\
& +\left(1-r_{1}\right) r_{2}\left(1-p_{b}\right) p(n+1,0,0,1) \\
& +\left(1-r_{1}\right) r_{b} p(n+1,0,1,0) \\
& +\left(1-r_{1}\right)\left(1-p_{2}\right)\left(1-p_{b}\right) p(n+1,0,1,1) \\
& +p_{1} r_{2}\left(1-p_{b}\right) p(n+1,1,0,1) \\
& +p_{1}\left(1-p_{2}\right)\left(1-p_{b}\right) p(n+1,1,1,1),
\end{aligned}
$$

$$
\begin{aligned}
p(n, 1,0,0) & \\
= & r_{1}\left(1-r_{2}\right)\left(1-r_{b}\right) p(n, 0,0,0) \\
& +r_{1}\left(1-r_{2}\right) p_{b} p(n, 0,0,1) \\
& +\left(1-r_{2}\right)\left(1-r_{b}\right) p(n, 1,0,0) \\
& +\left(1-r_{2}\right) p_{b} p(n, 1,0,1),
\end{aligned}
$$

$$
\begin{aligned}
p(n, 1,0,1) & \\
= & r_{1}\left(1-r_{2}\right) r_{b}(n-1,0,0,0) \\
& +r_{1}\left(1-r_{2}\right)\left(1-p_{b}\right) p(n-1,0,0,1) \\
& +r_{1} p_{2}\left(1-p_{b}\right) p(n-1,0,1,1) \\
& +\left(1-r_{2}\right) p_{b} p(n-1,1,0,0) \\
& +\left(1-p_{1}\right)\left(1-r_{2}\right)\left(1-p_{b}\right) p(n-1,1,0,1) \\
& +\left(1-p_{1}\right) p_{2}\left(1-p_{b}\right) p(n-1,1,1,1),
\end{aligned}
$$




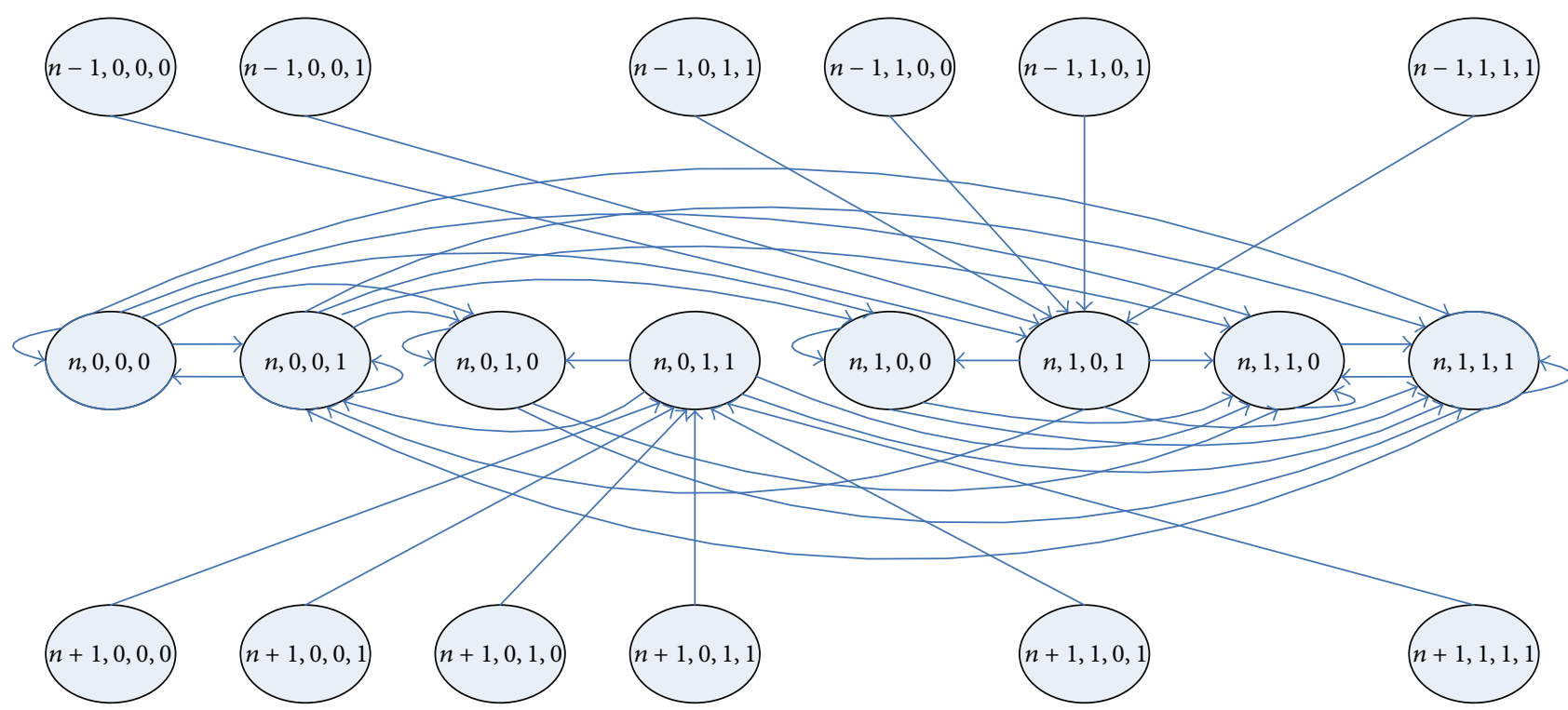

FIGURE 1: Internal state transitions.

$p(n, 1,1,0)$

$$
\begin{aligned}
= & r_{1} r_{2}\left(1-r_{b}\right) p(n, 0,0,0)+r_{1} r_{2} p_{b} p(n, 0,0,1) \\
& +r_{1}\left(1-r_{b}\right) p(n, 0,1,0)+r_{1} p_{b} p(n, 0,1,1) \\
& +r_{2}\left(1-r_{b}\right) p(n, 1,0,0)+r_{2} p_{b} p(n, 1,0,1) \\
& +\left(1-r_{b}\right) p(n, 1,1,0)+p_{b} p(n, 1,1,1),
\end{aligned}
$$

$p(n, 1,1,1)$

$$
\begin{aligned}
= & r_{1} r_{2} r_{b} p(n, 0,0,0)+r_{1} r_{2}\left(1-p_{b}\right) p(n, 0,0,1) \\
& +r_{1} r_{b} p(n, 0,1,0) \\
& +r_{1}\left(1-p_{2}\right)\left(1-p_{b}\right) p(n, 0,1,1) \\
& +r_{2} r_{b} p(n, 1,0,0) \\
& +\left(1-p_{1}\right) r_{2}\left(1-p_{b}\right) p(n, 1,0,1) \\
& +r_{b} p(n, 1,1,0) \\
& +\left(1-p_{1}\right)\left(1-p_{2}\right)\left(1-p_{b}\right) p(n, 1,1,1) .
\end{aligned}
$$

Then, the lower boundary state transitions are shown in Figure 2 and the lower boundary equations are derived:

$$
\begin{aligned}
p(0,0,0,0) & \\
= & \left(1-r_{1}\right)\left(1-r_{b}\right) p(0,0,1,0) \\
& +\left(1-r_{1}\right) p_{b} p(0,0,1,1) \\
p(0,0,1,1) & \\
= & \left(1-r_{1}\right) r_{b} p(0,0,1,0) \\
& +\left(1-r_{1}\right)\left(1-p_{b}\right) p(0,0,1,1)
\end{aligned}
$$

$+\left(1-r_{1}\right) r_{2} r_{b} p(1,0,0,0)$

$+\left(1-r_{1}\right) r_{2}\left(1-p_{b}\right) p(1,0,0,1)$

$+\left(1-r_{1}\right) r_{b} p(1,0,1,0)$

$+\left(1-r_{1}\right)\left(1-p_{2}\right)\left(1-p_{b}\right) p(1,0,1,1)$

$+p_{1}\left(1-p_{2}\right)\left(1-p_{b}\right) p(1,1,1,1)$,

$p(0,1,1,0)$

$$
\begin{aligned}
= & r_{1}\left(1-r_{b}\right) p(0,0,1,0)+r_{1} p_{b} p(0,0,1,1) \\
& +\left(1-r_{b}\right) p(0,1,1,0),
\end{aligned}
$$$$
p(1,0,0,0)
$$$$
=\left(1-r_{1}\right)\left(1-r_{2}\right)\left(1-r_{b}\right) p(1,0,0,0)
$$$$
+\left(1-r_{1}\right)\left(1-r_{2}\right) p_{b} p(1,0,0,1),
$$

$p(1,0,0,1)$

$=\left(1-r_{1}\right)\left(1-r_{2}\right) r_{b} p(1,0,0,0)$

$+\left(1-r_{1}\right)\left(1-r_{2}\right)\left(1-p_{b}\right) p(1,0,0,1)$

$+\left(1-r_{1}\right) p_{2}\left(1-p_{b}\right) p(1,0,1,1)$

$+p_{1} p_{2}\left(1-p_{b}\right) p(1,1,1,1)$,

$p(1,0,1,0)$

$$
\begin{aligned}
= & \left(1-r_{1}\right) r_{2}\left(1-r_{b}\right) p(1,0,0,0) \\
& +\left(1-r_{1}\right) r_{2} p_{b} p(1,0,0,1) \\
& +\left(1-r_{1}\right)\left(1-r_{b}\right) p(1,0,1,0) \\
& +\left(1-r_{1}\right) p_{b} p(1,0,1,1),
\end{aligned}
$$




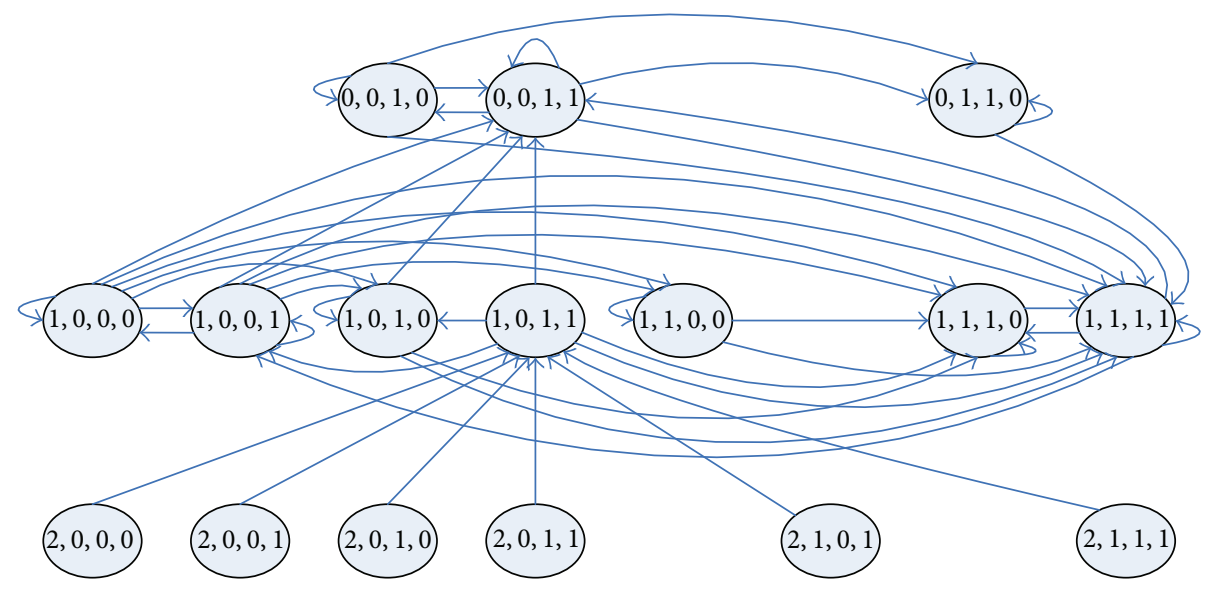

FIGURE 2: Lower boundary state transitions.

$$
\begin{aligned}
p(1,1,0,0) & \\
= & r_{1}\left(1-r_{2}\right)\left(1-r_{b}\right) p(1,0,0,0) \\
& +r_{1}\left(1-r_{2}\right) p_{b} p(1,0,0,1) \\
& +\left(1-r_{2}\right)\left(1-r_{b}\right) p(1,1,0,0), \\
p(1,1,1,0) & \\
= & r_{1} r_{2}\left(1-r_{b}\right) p(1,0,0,0)+r_{1} r_{2} p_{b} p(1,0,0,1) \\
& +r_{1}\left(1-r_{b}\right) p(1,0,1,0)+r_{1} p_{b} p(1,0,1,1) \\
& +r_{2}\left(1-r_{b}\right) p(1,1,0,0)+\left(1-r_{b}\right) p(1,1,1,0) \\
& +p_{b} p(1,1,1,1), \\
p(1, & 1,1,1) \\
= & r_{1} r_{b} p(0,0,1,0)+r_{1}\left(1-p_{b}\right) p(0,0,1,1) \\
& +r_{b} p(0,1,1,0)+r_{1} r_{2} r_{b} p(1,0,0,0) \\
& +r_{1} r_{2}\left(1-p_{b}\right) p(1,0,0,1)+r_{1} r_{b} p(1,0,1,0) \\
& +r_{1}\left(1-p_{2}\right)\left(1-p_{b}\right) p(1,0,1,1) \\
& +r_{2} r_{b} p(1,1,0,0)+r_{b} p(1,1,1,0) \\
& +\left(1-p_{1}\right)\left(1-p_{2}\right)\left(1-p_{b}\right) p(1,1,1,1) .
\end{aligned}
$$

Finally, Figure 3 illustrates the upper boundary state transitions and the following are the upper boundary equations:

$$
\begin{aligned}
p(N, 1,0,0) & \\
= & \left(1-r_{2}\right)\left(1-r_{b}\right) p(N, 1,0,0) \\
& +\left(1-r_{2}\right) p_{b} p(N, 1,0,1),
\end{aligned}
$$

$p(N, 1,0,1)$

$$
=\left(1-r_{2}\right) r_{b} p(N, 1,0,0)
$$

$$
\begin{aligned}
& +\left(1-r_{2}\right)\left(1-p_{b}\right) p(N, 1,0,1) \\
& +r_{1}\left(1-r_{2}\right) r_{b} p(N-1,0,0,0) \\
& +r_{1}\left(1-r_{2}\right)\left(1-p_{b}\right) p(N-1,0,0,1) \\
& +\left(1-r_{2}\right) r_{b} p(N-1,1,0,0) \\
& +\left(1-p_{1}\right)\left(1-r_{2}\right)\left(1-p_{b}\right) p(N-1,1,0,1) \\
& +\left(1-p_{1}\right) p_{2}\left(1-p_{b}\right) p(N-1,1,1,1), \\
& p(N, 1,1,0) \\
& =r_{2}\left(1-r_{b}\right) p(N, 1,0,0)+r_{2} p_{b} p(N, 1,0,1) \\
& +\left(1-r_{b}\right) p(N, 1,1,0), \\
& p(N-1,0,0,0) \\
& =\left(1-r_{1}\right)\left(1-r_{2}\right)\left(1-r_{b}\right) p(N-1,0,0,0) \\
& +\left(1-r_{1}\right)\left(1-r_{2}\right) p_{b} p(N-1,0,0,1), \\
& p(N-1,0,0,1) \\
& =\left(1-r_{1}\right)\left(1-r_{2}\right) r_{b} p(N-1,0,0,0) \\
& +\left(1-r_{1}\right)\left(1-r_{2}\right)\left(1-p_{b}\right) p(N-1,0,0,1) \\
& +p_{1}\left(1-r_{2}\right)\left(1-p_{b}\right) p(N-1,1,0,1) \\
& +p_{1} p_{2}\left(1-p_{b}\right) p(N-1,1,1,1), \\
& p(N-1,0,1,0) \\
& =\left(1-r_{1}\right) r_{2}\left(1-r_{b}\right) p(N-1,0,0,0) \\
& +\left(1-r_{1}\right) r_{2} p_{b} p(N-1,0,0,1) \\
& +\left(1-r_{1}\right)\left(1-r_{b}\right) p(N-1,0,1,0), \\
& p(N-1,1,0,0) \\
& =r_{1}\left(1-r_{2}\right)\left(1-r_{b}\right) p(N-1,0,0,0)
\end{aligned}
$$




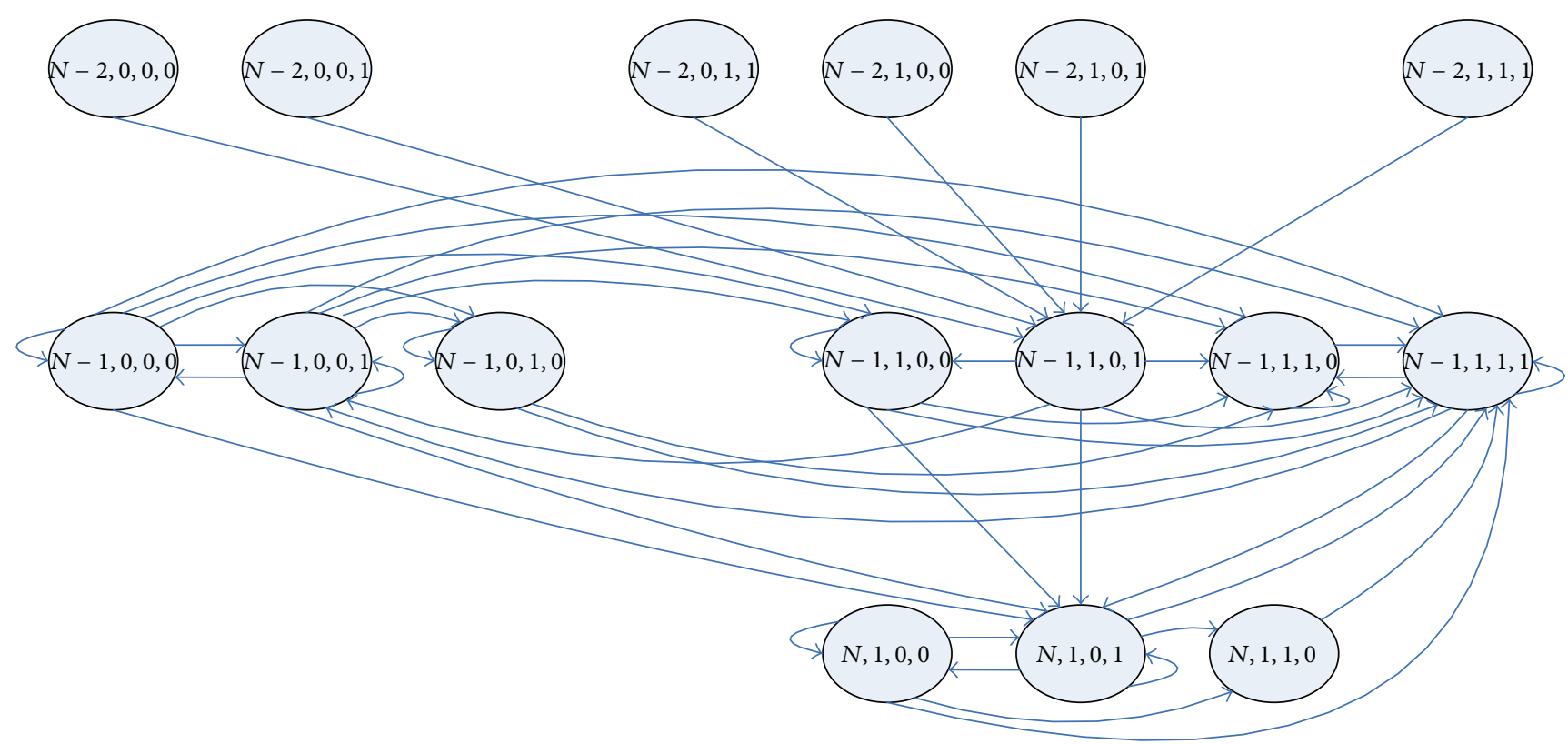

FIGURE 3: Upper boundary state transitions.

$$
\begin{aligned}
& +r_{1}\left(1-r_{2}\right) p_{b} p(N-1,0,0,1) \\
& +\left(1-r_{2}\right)\left(1-r_{b}\right) p(N-1,1,0,0) \\
& +\left(1-r_{2}\right) p_{b} p(N-1,1,0,1), \\
p(N- & 1,1,1,0) \\
= & r_{1} r_{2}\left(1-r_{b}\right) p(N-1,0,0,0) \\
& +r_{1} r_{2} p_{b} p(N-1,0,0,1) \\
& +r_{1}\left(1-r_{b}\right) p(N-1,0,1,0) \\
& +r_{2}\left(1-r_{b}\right) p(N-1,1,0,0) \\
& +r_{2} p_{b} p(N-1,1,0,1) \\
& +\left(1-r_{b}\right) p(N-1,1,1,0) \\
& +p_{b} p(N-1,1,1,1), \\
p(N- & 1,1,1,1) \\
= & r_{2} r_{b} p(N, 1,0,0)+r_{2}\left(1-p_{b}\right) p(N, 1,0,1) \\
& +r_{b} p(N, 1,1,0)+r_{1} r_{2} r_{b} p(N-1,0,0,0) \\
& +r_{1} r_{2}\left(1-p_{b}\right) p(N-1,0,0,1) \\
& +r_{1} r_{b} p(N-1,0,1,0)+r_{2} r_{b} p(N-1,1,0,0) \\
& +\left(1-p_{1}\right) r_{2}\left(1-p_{b}\right) p(N-1,1,0,1) \\
& +r_{b} p(N-1,1,1,0) \\
& +\left(1-p_{1}\right)\left(1-p_{2}\right)\left(1-p_{b}\right) p(N-1,1,1,1) .
\end{aligned}
$$

3.3. Solution of the Equations. First, the internal equations are solved. Markov process is a birth-death process where the state transitions are of only two types: "births," which increase the state variable by 1 , and "deaths," which decrease the state by 1 . When deriving the transition equations, it can be observed that the buffer level can increase or decrease only by 1 between two consecutive time units. From Figure 1 we can know that $(n, 0,1,1)$ is the only one which can be reached from states with a buffer level of $n+1$ and, by substituting $n+1$ for $n,(n+1,1,0,1)$ is the only state which can be reached from states with a buffer level of $n$. Correspondingly, (18) gives the probability of a state with a buffer level of $n+1$ becoming a state with buffer level of $n$, which is called death rate. By substituting $n+1$ for $n$, (20) gives the probability of a state with a buffer level of $n$ becoming a state with buffer level of $n+1$, which is called birth rate. In a steady birth-death process, the rate of transitions in both directions must be equal; that is, the birth rate and the death rate should be identical. Therefore, the following equation is obtained:

$$
p(n, 0,1,1)=p(n+1,1,0,1) .
$$

By substituting $n-1$ for $n$ in (18), substituting $n+1$ for $n$ in (20) and using (25), we have

$$
\begin{aligned}
& p(n, 1,0,1) \\
&=\left(1-r_{1}\right) r_{2} r_{b} p(n, 0,0,0) \\
&+\left(1-r_{1}\right) r_{2}\left(1-p_{b}\right) p(n, 0,0,1) \\
&+\left(1-r_{1}\right) r_{b} p(n, 0,1,0) \\
&+\left(1-r_{1}\right)\left(1-p_{2}\right)\left(1-p_{b}\right) p(n, 0,1,1)
\end{aligned}
$$




$$
\begin{aligned}
& +p_{1} r_{2}\left(1-p_{b}\right) p(n, 1,0,1) \\
& +p_{1}\left(1-p_{2}\right)\left(1-p_{b}\right) p(n, 1,1,1), \\
p(n, 0,1,1) & \\
= & r_{1}\left(1-r_{2}\right) r_{b}(n, 0,0,0) \\
& +r_{1}\left(1-r_{2}\right)\left(1-p_{b}\right) p(n, 0,0,1) \\
& +r_{1} p_{2}\left(1-p_{b}\right) p(n, 0,1,1) \\
& +\left(1-r_{2}\right) p_{b} p(n, 1,0,0) \\
& +\left(1-p_{1}\right)\left(1-r_{2}\right)\left(1-p_{b}\right) p(n, 1,0,1) \\
& +\left(1-p_{1}\right) p_{2}\left(1-p_{b}\right) p(n, 1,1,1) .
\end{aligned}
$$

Equations (15)-(17), (19), (21), (22), and (26) form a homogeneous equation set with 8 unknown parameters in which all involved states have the same buffer level $n$. By observing the equation set, we find that there is only one basic solution for this equation set. For $n=2, \ldots, N-2$, all the equation sets have the same basic solution. Let $p(n, 1,1,1)=$ 1 ; solve the equation set and we can get the basic solution

$$
\begin{gathered}
\phi=(\phi(0,0,0), \phi(0,0,1), \phi(0,1,0), \phi(0,1,1), \\
\phi(1,0,0), \phi(1,0,1), \phi(1,1,0), \phi(1,1,1)),
\end{gathered}
$$

where $\phi(1,1,1)=1$

For the equation set with buffer level $n$, the solution is denoted as $\phi_{n}$. Let $K_{n}$ be the ratio of $\phi_{n}$ to $\phi$. We have

$$
\begin{aligned}
& \phi_{n}=(p(n, 0,0,0), p(n, 0,0,1), p(n, 0,1,0), \\
& \quad p(n, 0,1,1), p(n, 1,0,0), p(n, 1,0,1), p(n, 1,1,0), \\
& p(n, 1,1,1))=K_{n}(\phi(0,0,0), \phi(0,0,1), \phi(0,1,0), \\
& \phi(0,1,1), \phi(1,0,0), \phi(1,0,1), \phi(1,1,0), \\
& \phi(1,1,1))=K_{n} \phi .
\end{aligned}
$$

By substituting (28) into (18), we obtain

$$
\begin{aligned}
K_{n} \phi( & 0,1,1) \\
= & \left(1-r_{1}\right) r_{2} r_{b} K_{n+1} \phi(0,0,0) \\
& +\left(1-r_{1}\right) r_{2}\left(1-p_{b}\right) K_{n+1} \phi(0,0,1) \\
& +\left(1-r_{1}\right) r_{b} K_{n+1} \phi(0,1,0) \\
& +\left(1-r_{1}\right)\left(1-p_{2}\right)\left(1-p_{b}\right) K_{n+1} \phi(0,1,1) \\
& +p_{1} r_{2}\left(1-p_{b}\right) K_{n+1} \phi(1,0,1) \\
& +p_{1}\left(1-p_{2}\right)\left(1-p_{b}\right) K_{n+1} \phi(1,1,1) .
\end{aligned}
$$

Let $X_{n}$ be the ratio of $K_{n}$ to $K_{n+1}$, and according to (29) the following equation is derived:

$$
\begin{aligned}
X_{n}= & \frac{K_{n}}{K_{n+1}} \\
= & \left(1-r_{1}\right) r_{2} r_{b} \frac{\phi(0,0,0)}{\phi(0,1,1)} \\
& +\left(1-r_{1}\right) r_{2}\left(1-p_{b}\right) \frac{\phi(0,0,1)}{\phi(0,1,1)} \\
& +\left(1-r_{1}\right) r_{b} \frac{\phi(0,1,0)}{\phi(0,1,1)} \\
& +\left(1-r_{1}\right)\left(1-p_{2}\right)\left(1-p_{b}\right) \frac{\phi(0,1,1)}{\phi(0,1,1)} \\
& +p_{1} r_{2}\left(1-p_{b}\right) \frac{\phi(1,0,1)}{\phi(0,1,1)} \\
& +p_{1}\left(1-p_{2}\right)\left(1-p_{b}\right) \frac{\phi(1,1,1)}{\phi(0,1,1)} .
\end{aligned}
$$

From this equation, it should be noticed that $X_{n}=X$ is constant and is independent of $n$. Thus,

$$
\phi_{n}=X^{-1} \phi_{n-1}=X^{-n+2} K_{2} \phi \text {. }
$$

Since $K_{2}$ is constant, let $C=X^{2} K_{2}$ and we have

$$
\phi_{n}=C X^{-n} \phi
$$

Now we show the solution of the boundary equations. As shown in Figure 2, the state $(1,1,0,1)$ can be treated as an internal state because it is only reached from the states with a buffer level of 2 , so that

$$
p(1,1,0,1)=C X^{-1} \phi(1,0,1) .
$$

According to (23), the probabilities of other lower boundary states can be calculated.

Similarly, for the upper boundary states, $(N-1,1,0,1)$ can be treated as an internal state and its probability is

$$
p(N-1,1,0,1)=C X^{-N+1} \phi(1,0,1) \text {. }
$$

The probabilities of other upper boundary states can be given according to (24).

Finally, these equations have to satisfy the normalization equation

$$
1=\sum_{n=0}^{N} \sum_{\substack{\alpha_{1}=0,1 \\ \alpha_{2}=0,1 \\ \alpha_{b}=0,1}} p\left(n, \alpha_{1}, \alpha_{2}, \alpha_{b}\right)
$$

The unknown constant $C$ is calculated and so are other unknown parameters. The production rate and the average buffer level can be obtained from (12) and (14). 
TABLE 1: Configurations of cases.

\begin{tabular}{lccc}
\hline & Case 1 & Case 2 & Case 3 \\
\hline$p_{1}$ & 0.005 & 0.001 & 0.005 \\
$r_{1}$ & 0.05 & 0.05 & 0.05 \\
$p_{2}$ & 0.001 & 0.005 & 0.005 \\
$r_{2}$ & 0.05 & 0.05 & 0.05 \\
$p_{b}$ & Varied & Varied & Varied \\
$r_{b}$ & 0.008 & 0.008 & 0.008 \\
\hline
\end{tabular}

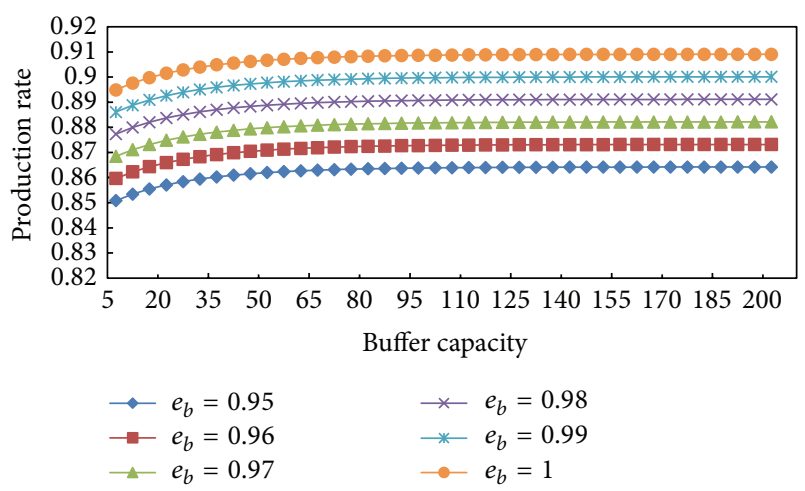

FIGURE 4: Production rate for different combinations of buffer capacity and isolated efficiency (Case 1).

\section{Numerical Cases}

The developed method is validated in this section. In Section 4.1, numerical cases were generated to analyze the effects of buffer reliability on the production rate and the average buffer level. In Section 4.2, buffer subject to time-dependent failure is compared with buffer subject to operation-dependent failure.

4.1. Buffer Reliability Effects. Three representative cases in [23] were chosen to analyze the effects. In Case 1, the two machines are relatively reliable and the isolated efficiency of the first machine is about $10 \%$ higher than the one of the second machine. After switching the two machines in Case 1, we have Case 2. In Case 3, the two machines are identical. Table 1 illustrates a list of parameters for these cases. For the buffers in all cases, the repair rates are equal to 0.008 and the buffer reliabilities increase from 0.95 to 1 . The failure rates of the buffers are calculated based on the repair rates and the buffer reliabilities. The buffer capacities are varied from 5 to 200.

Figures 4, 5, and 6 show the effects of the buffer reliability on the production rate. According to these figures, the production rate decreases along with the decrease of the buffer reliability which indicates the detrimental impact of low buffer reliability. When the buffer reliability is fixed, the production rate increases and reaches a limit when the buffer capacity increases from 5 to 200.

Figures 7, 8, and 9 show the effects of the buffer reliability on the average buffer level. In general, when the buffer reliability or the buffer capacity increases, the average buffer



FIGURE 5: Production rate for different combinations of buffer capacity and isolated efficiency (Case 2).

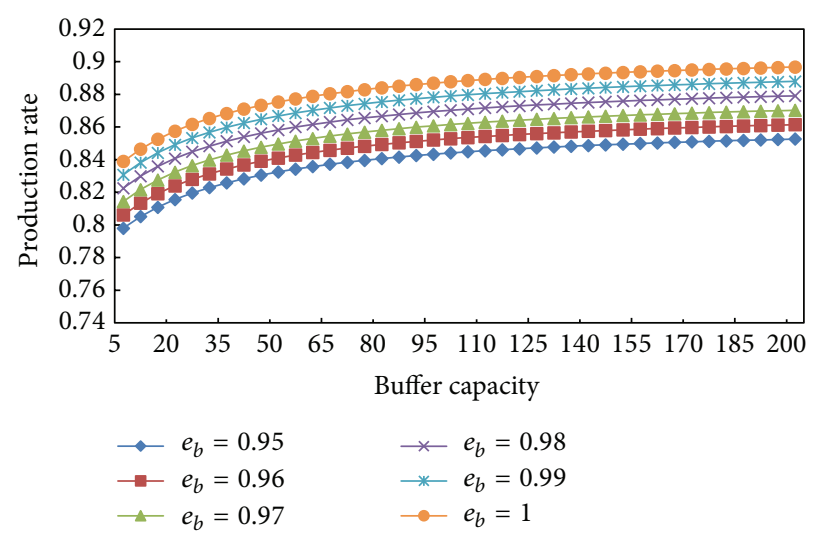

Figure 6: Production rate for different combinations of buffer capacity and isolated efficiency (Case 3).

level grows. In Case 1, as machine $M_{1}$ is less efficient than machine $M_{2}$, the average buffer level approaches a limit when the buffer capacity increases. On the contrary, in Case 2, in which the isolated efficiency of machine $M_{2}$ is lower than the one of machine $M_{1}$, the limit of the average buffer level does not exist.

The results above also prove the reversibility of manufacturing systems, a phenomenon discovered by Ammar and Gershwin [28]. As Case 2 is a reversed system of Case 1, the production rates of corresponding systems in Case 1 and Case 2 are identical and the sums of the average buffer levels are equal to the buffer capacities. Therefore, as the parameters of machine $M_{1}$ and machine $M_{2}$ in Case 3 are the same, the average buffer levels of Case 3 are always half of the buffer capacities despite the variation of the buffer capacity.

4.2. Comparisons. A case is used to compare the two different types of buffers. The failure rates of machine $M_{1}$ and machine $M_{2}$ are 0.005 and 0.001 , respectively. The repair rates of the two machines are both 0.05 . Hence, machine $M_{1}$ is less efficient than machine $M_{2}$. The buffer capacity is 5. The repair rate of the buffer is 0.008 . The isolated efficiency of the buffer is varied from 1 to 0.1 . The results of the production rate are shown in Figure 10 and the results of the average buffer 


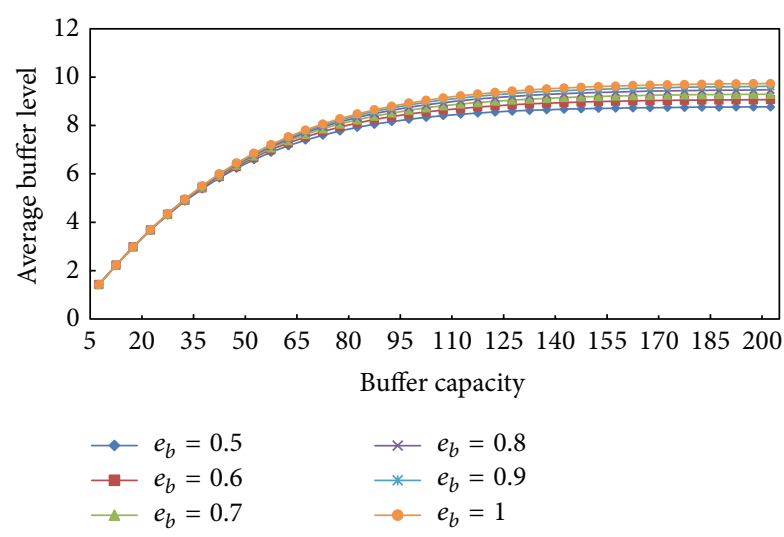

Figure 7: Average buffer level for different combinations of buffer capacity and isolated efficiency (Case 1).

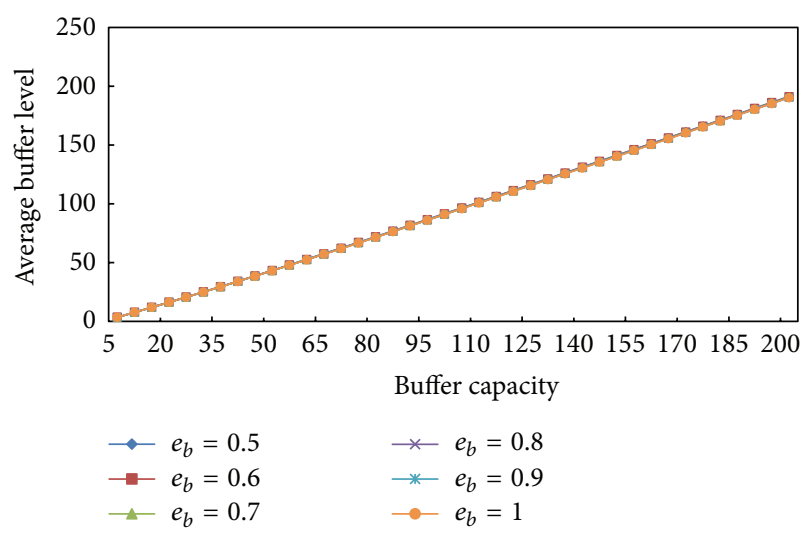

Figure 8: Average buffer level for different combinations of buffer capacity and isolated efficiency (Case 2).

level are shown in Figure 11. When the buffer is reliable, that is, $e_{b}=1$, the production rates are the same. So are the average buffer levels. When it is unreliable, the buffer subject to time-dependent failure causes more loss in production rate than the buffer subject to operation-dependent failure. The average buffer level of the former one is also lower than the average buffer level of the latter one. It is quite reasonable since a buffer subject to time-dependent failure, such as an accumulating conveyor, can break down even when there is no part on it, which results in the growth of the blockage probability of machine $M_{1}$. According to the reversibility of manufacturing systems, it can be inferred that if the isolated efficiencies of machine $M_{1}$ and machine $M_{2}$ are identical the production rates of the systems with the two types of buffers still act in the same way while the buffer levels are identical.

\section{Conclusions and Future Work}

In this paper, a two-machine manufacturing system with a finite buffer subject to time-dependent failure is analyzed. The representative of this type of buffers is accumulating conveyor which is widely used in reality. As far as we know, only one method has been proposed to study the behaviour



Figure 9: Average buffer level for different combinations of buffer capacity and isolated efficiency (Case 3).

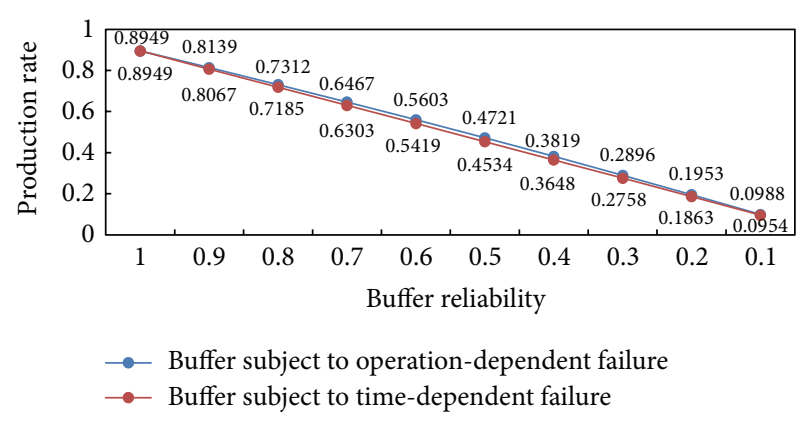

Figure 10: Comparison of the production rates generated by two different types of buffer.

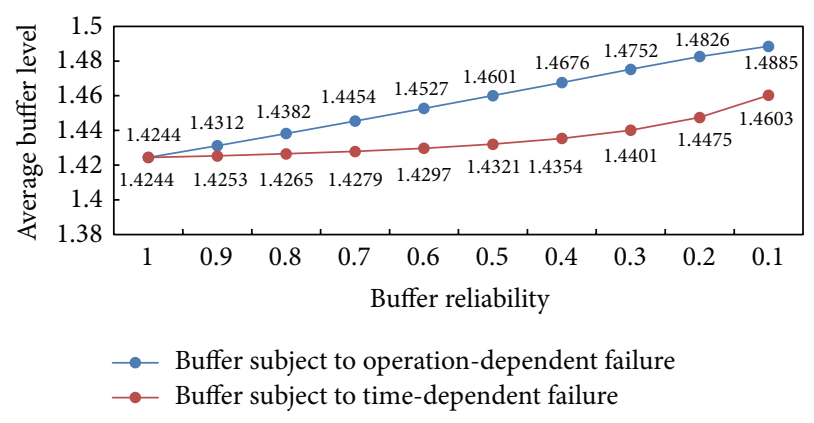

FIGURE 11: Comparison of the average buffer levels generated by two different types of buffer.

of this type of buffers. However, it is an approximate one. The main contribution of this paper is the development of an exact analytical method. A Markov model is established for the system. Then transition equations are derived and solved. Numerical experiments are implemented to investigate the relationships between buffer parameters and system performance. Also, the difference between buffer subject to timedependent failure and buffer subject to operation-dependent failure is discussed. As the building block of approximate analytical methods for the analysis of large systems, the proposed method is of great significance. 
Future work would be devoted to proposing a decomposition method for evaluating large systems and developing an exact analytical method for the analysis of inhomogeneous two-machine systems based on continuous Markov modelling techniques.

\section{Conflict of Interests}

The authors declare that there is no conflict of interests regarding the publication of this paper.

\section{Acknowledgment}

This project is supported by National Natural Science Foundation of China (Grant nos. 71401098 and 51205242).

\section{References}

[1] D. Battini, A. Persona, and A. Regattieri, "Buffer size design linked to reliability performance: a simulative study," Computers \& Industrial Engineering, vol. 56, no. 4, pp. 1633-1641, 2009.

[2] J. A. Buzacott and L. E. Hanifin, "Models of automatic transfer lines with inventory banks a review and comparison," A I I E Transactions, vol. 10, no. 2, pp. 197-207, 2007.

[3] Y. Dallery, R. David, and X.-L. Xie, "Approximate analysis of transfer lines with unreliable machines and finite buffers," IEEE Transactions on Automatic Control, vol. 34, no. 9, pp. 943-953, 1989.

[4] R. Lipset, R. P. Van Til, and S. Sengupta, "Development of a model for a two-station serial transfer line subject to machine and buffer failure," Mathematical and Computer Modelling of Dynamical Systems, vol. 4, no. 3, pp. 231-246, 1998.

[5] Y. Dallery and H. Le Bihan, "An improved decomposition method for the analysis of production lines with unreliable machines and finite buffers," International Journal of Production Research, vol. 37, no. 5, pp. 1093-1117, 1999.

[6] Y. Dallery and S. B. Gershwin, "Manufacturing flow line systems: a review of models and analytical results," Queueing Systems, vol. 12, no. 1-2, pp. 3-94, 1992.

[7] J. A. Buzacott, "Automatic transfer lines with buffer stocks," International Journal of Production Research, vol. 5, no. 3, pp. 183-200, 1967.

[8] F. S. Hillier and R. W. Boling, "Finite queues in series with exponential or erlang service times-a numerical approach," Operations Research, vol. 15, no. 2, pp. 286-303, 1967.

[9] T. Altiok and S. Stidham Jr., "The allocation of interstage buffer capacities in production lines," AIIE Transactions, vol. 15, no. 4, pp. 292-299, 1983.

[10] B. Zimmern, "Ètudes de la propagation des arrêsts aléatoires dans les chaines de production," Revue de Statistique Appliquée, vol. 4, no. 1, pp. 85-104, 1956.

[11] S. B. Gershwin and I. C. Schick, Continuous Model of an Unreliable Two-Stage Material Flow System with a Finite Interstagebuffer, Massachusetts Institute of Technology, Cambridge, Mass, USA, 1980.

[12] D. Dubois and J. Forestier, "Productivité et en-cours moyens d'un ensemble de deux machines séparées par une zone de stockage," RAIRO Automatique, vol. 16, no. 2, pp. 105-132, 1982.

[13] S. Yeralan, W. E. Franck, and M. A. Quasem, "A continuous materials flow production line model with station breakdown,"
European Journal of Operational Research, vol. 27, no. 3, pp. 289300, 1986.

[14] S. Yeralan and B. Tanj, "A station model for continuous materials flow production systems," International Journal of Production Research, vol. 35, no. 9, pp. 2525-2541, 1997.

[15] T. Tolio, A. Matta, and S. B. Gershwin, "Analysis of two-machine lines with multiple failure modes," IIE Transactions, vol. 34, no. 1, pp. 51-62, 2002.

[16] B. Tan and S. B. Gershwin, "Analysis of a general Markovian two-stage continuous-flow production system with a finite buffer," International Journal of Production Economics, vol. 120, no. 2, pp. 327-339, 2009.

[17] B. Tan and S. B. Gershwin, "Modelling and analysis of Markovian continuous flow systems with a finite buffer," Annals of Operations Research, vol. 182, no. 1, pp. 5-30, 2011.

[18] N. Li, Z. Jiang, G. Liu, and Z. Zhang, "Analysis of qualitycaused re-entrance electrical test system in semiconductor manufacturing by Markov method," International Journal of Production Research, vol. 50, no. 12, pp. 3486-3497, 2012.

[19] E. Gebennini and S. B. Gershwin, "Modeling waste production into two-machine-one-buffer transfer lines," IIE Transactions, vol. 45, no. 6, pp. 591-604, 2013.

[20] E. Gebennini, A. Grassi, C. Fantuzzi, S. B. Gershwin, and I. C. Schick, "Discrete time model for two-machine one-buffer transfer lines with restart policy," Annals of Operations Research, vol. 209, no. 1, pp. 41-65, 2013.

[21] E. Gebennini, A. Grassi, and C. Fantuzzi, "The two-machine one-buffer continuous time model with restart policy," Annals of Operations Research, 2013.

[22] J. Li, D. E. Blumenfeld, N. Huang, and J. M. Alden, “Throughput analysis of production systems: recent advances and future topics," International Journal of Production Research, vol. 47, no. 14, pp. 3823-3851, 2009.

[23] M. H. Burman, New results in flow line analysis [Ph.D. thesis], Massachusetts Institute of Technology, Cambridge, Mass, USA, 1995.

[24] S. M. A. Suliman, "A mathematical model for a buffered twostage manufacturing cell with an unreliable transfer device," International Journal of Production Economics, vol. 63, no. 1, pp. 69-81, 2000.

[25] J. Liu, Z. Y. Rui, R. C. Feng, and C. L. Lei, "Performance evaluation of hybrid production lines with multiple stochastic failure modes and unreliable limited buffers," Applied Mechanics and Materials, vol. 58-60, pp. 410-416, 2011.

[26] R. Lipset, S. Sengupta, and R. P. Van Til, "Analytic modeling of the unreliable buffer in the serial transfer line," in Proceedings of the 26th Southeastern Symposium on System Theory, pp. 122-126, Athens, Ga, USA, March 1994.

[27] R. Lipset, R. P. Van Til, and S. Sengupta, "Steady-state performance analysis of serial transfer lines subject to machine and buffer failure," IEEE Transactions on Automatic Control, vol. 44, no. 2, pp. 319-325, 1999.

[28] M. H. Ammar and S. B. Gershwin, "Equivalence relations in queueing models of fork/join networks with blocking," Performance Evaluation, vol. 10, no. 3, pp. 233-245, 1989. 


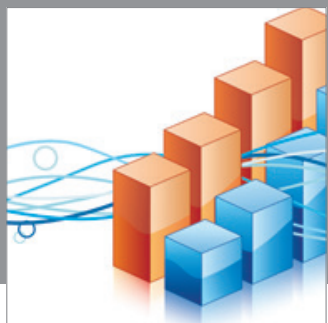

Advances in

Operations Research

mansans

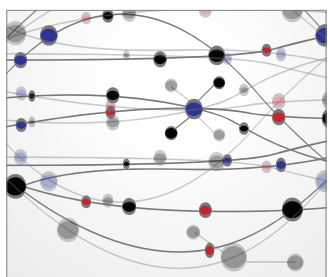

The Scientific World Journal
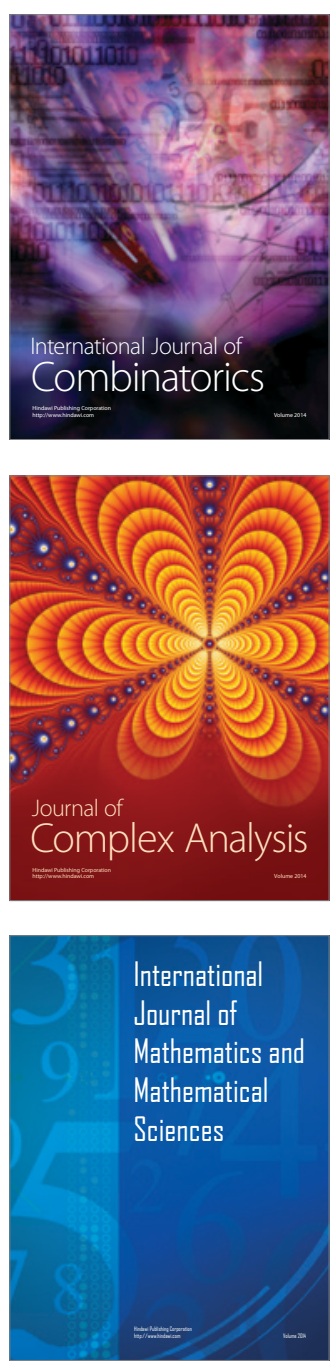
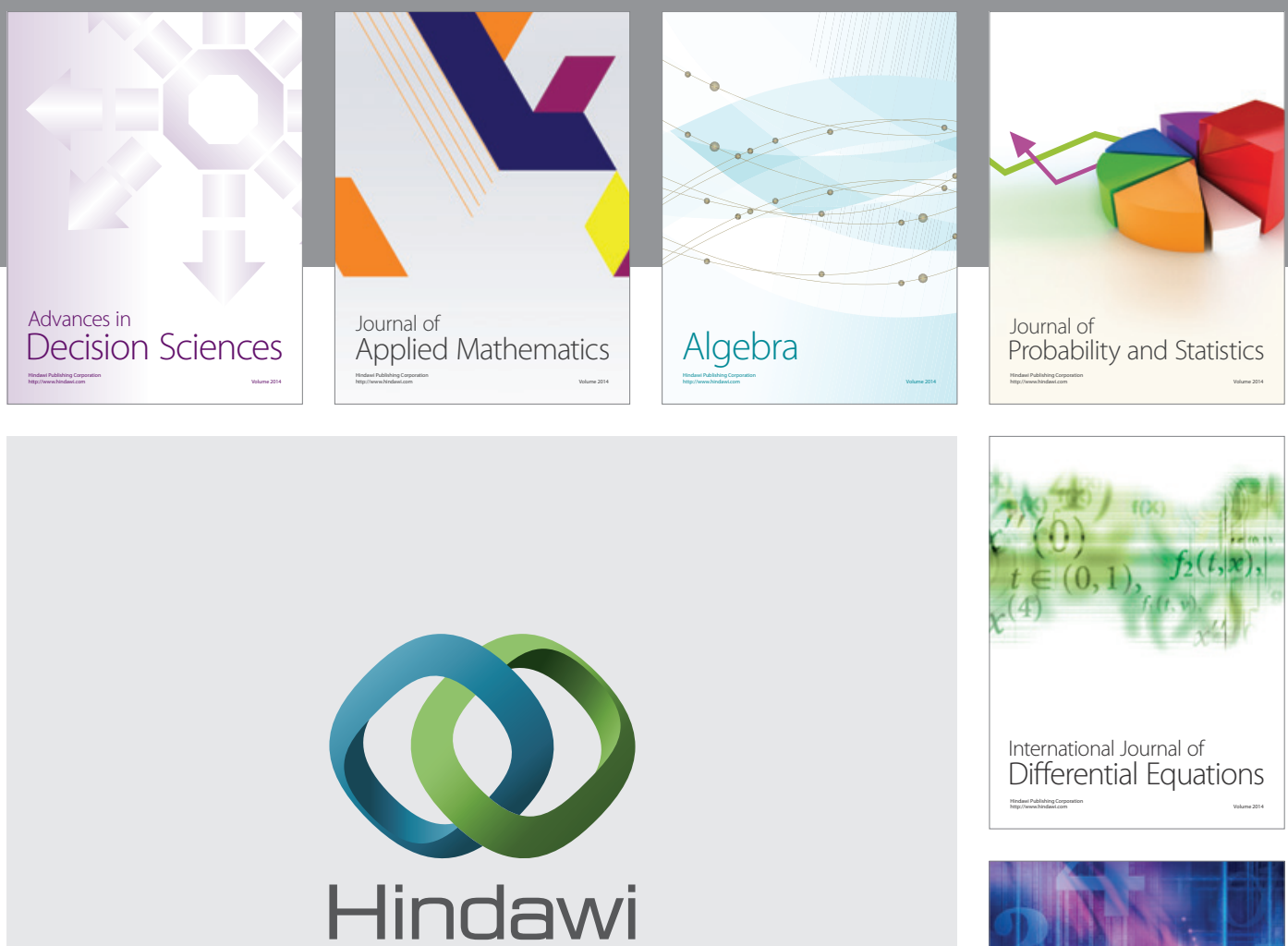

Submit your manuscripts at http://www.hindawi.com
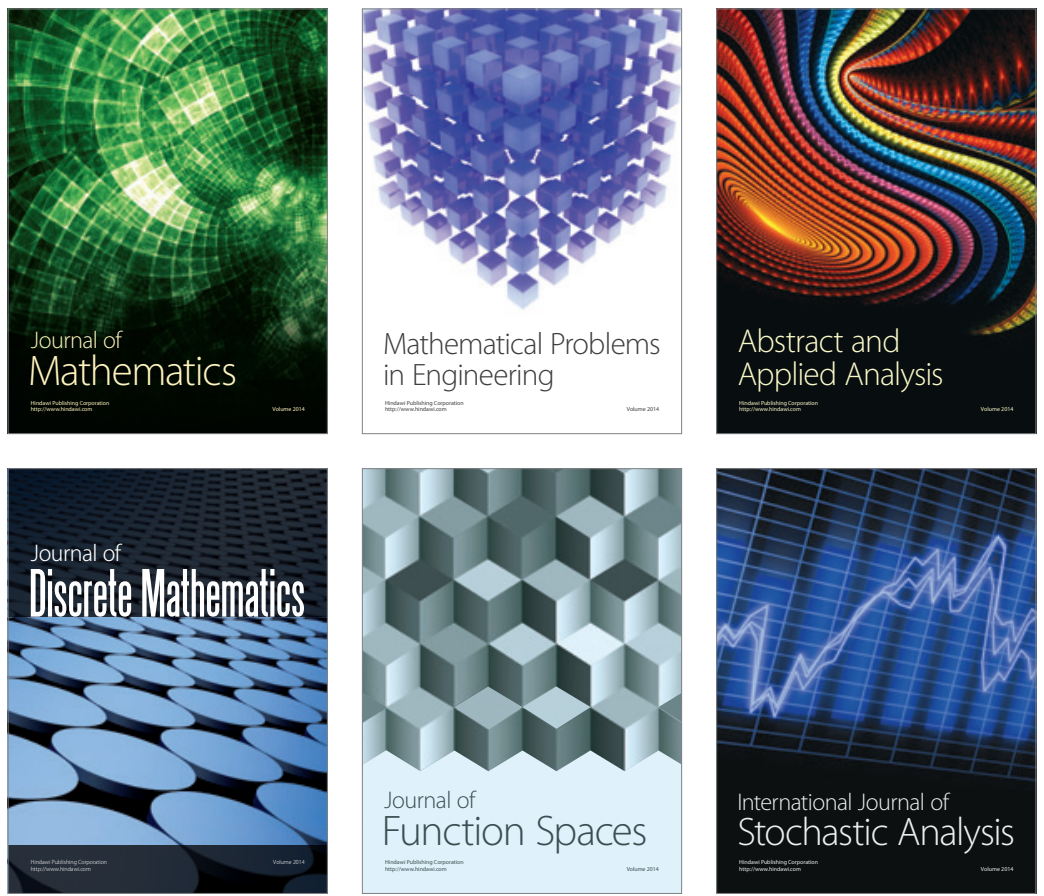

Journal of

Function Spaces

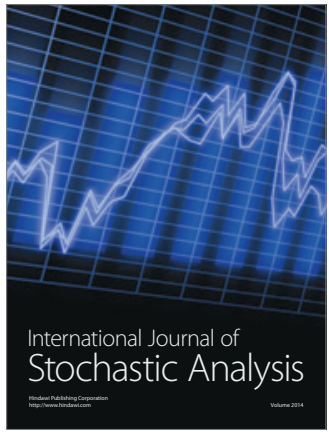

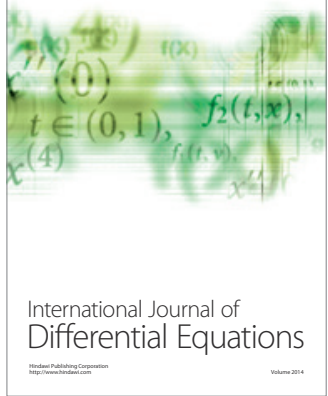
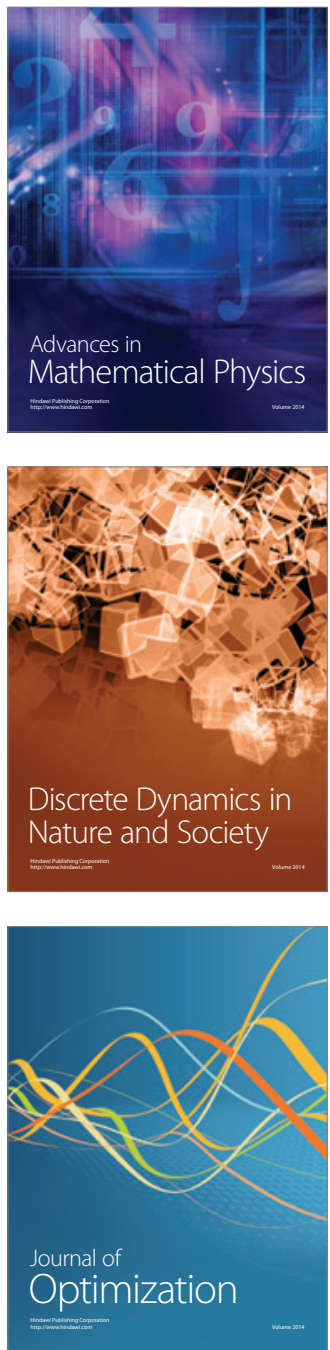